\title{
Enhancing 3-hydroxypropionic acid production in combination with sugar supply engineering by cell surface-display and metabolic engineering of Schizosaccharomyces pombe
}

Seiya Takayama ${ }^{1}$, Aiko Ozaki ${ }^{1}$, Rie Konishi ${ }^{2}$, Chisako Otomo², Mayumi Kishida², Yuuki Hirata²,

Takuya Matsumoto ${ }^{2}$, Tsutomu Tanaka ${ }^{1 *}$ (D) and Akihiko Kondo ${ }^{2}$

\begin{abstract}
Background: Economical production of value-added chemicals from renewable biomass is a promising path to sustainability. 3-Hydroxypropionic acid (3-HP) is an important chemical for building a bio-sustainable society. Establishment of 3-HP production from renewable resources such as glucose would provide a bio-sustainable alternative to the production of acrylic acid from fossil resources.

Results: Here, we describe metabolic engineering of the fission yeast Schizosaccharomyces pombe to enhance 3-HP production from glucose and cellobiose via the malonyl-CoA pathway. The mcr gene, encoding the malonyl-CoA reductase of Chloroflexus aurantiacus, was dissected into two functionally distinct fragments, and the activities of the encoded protein were balanced. To increase the cellular supply of malonyl-CoA and acetyl-CoA, we introduced genes encoding endogenous aldehyde dehydrogenase, acetyl-CoA synthase from Salmonella enterica, and endogenous pantothenate kinase. The resulting strain produced 3-HP at $1.0 \mathrm{~g} / \mathrm{L}$ from a culture starting at a glucose concentration of $50 \mathrm{~g} / \mathrm{L}$. We also engineered the sugar supply by displaying beta-glucosidase (BGL) on the yeast cell surface. When grown on $50 \mathrm{~g} / \mathrm{L}$ cellobiose, the beta-glucosidase-displaying strain consumed cellobiose efficiently and produced 3-HP at $3.5 \mathrm{~g} / \mathrm{L}$. Under fed-batch conditions starting from cellobiose, this strain produced 3-HP at up to $11.4 \mathrm{~g} / \mathrm{L}$, corresponding to a yield of $11.2 \%$ (g-3-HP/g-glucose; given that $1 \mathrm{~g}$ cellobiose corresponds to $1.1 \mathrm{~g}$ glucose upon digestion).
\end{abstract}

Conclusions: In this study, we constructed a series of S. pombe strains that produced 3-HP via the malonyl-CoA pathway. Our study also demonstrated that BGL display using cellobiose and/or cello-oligosaccharides as a carbon source has the potential to improve the titer and yield of malonyl-CoA- and acetyl-CoA-derived compounds.

Keywords: Schizosaccharomyces pombe, 3-Hydroxypropionic acid, CRISPR-Cas9 system, Cell surface display, Malonyl$\mathrm{COA}$

\footnotetext{
*Correspondence: tanaka@kitty.kobe-u.ac.jp

${ }^{1}$ Department of Chemical Science and Engineering, Graduate School of Engineering, Kobe University, 1-1 Rokkodaicho, Nada, Kobe 657-8501,

Japan

Full list of author information is available at the end of the article
} 


\section{Background}

3-Hydoroxypropionic acid (3-HP) is a platform chemical that serves as a precursor to various valuable chemicals, including acrylic acid, acrylonitrile, and 1,3-propanediol. Acrylic acid, which is used as a super absorbent polymer and in plastic manufacturing, currently is produced primarily by catalytic oxidation of petroleum-derived propane. Acrylonitrile, a compound that is produced at more than 14 billion pounds per year, is used to generate plastics, resins, and fibers [1]. Hence, the US Department of Energy has designated 3-HP as one of the most important substances that should be targeted for production from renewable resources [2]. Establishment of 3-HP production from renewable resources such as glucose would provide a bio-sustainable alternative to the production of acrylic acid from fossil resources.

Microbial bioproduction is one of the best solutions for building a sustainable economy. Four pathways (including those employing glycerol, lactate, malonyl-CoA, or $\beta$-alanine) have been defined for the production of 3-HP in vivo using microorganisms. Klebsiella pneumoniae and Lactobacillus reutei are known to produce 3-HP from glycerol [3]. The mechanism of 3-HP production from glycerol consists of 2 steps. First, glycerol is converted to 3-hydroxypropionaldehyde (3-HPA) via a reaction catalyzed by a coenzyme vitamin B12-dependent glycerol dehydratase. Next, 3-HPA is converted to 3-HP via a reaction catalyzed by aldehyde dehydrogenase [47]. This route is not suitable for industrial use, because typical host production microorganisms [notably, E. coli and budding yeast (Saccharomyces cerevisiae)] cannot synthesize vitamin B12; such host organisms therefore would require a continuous exogenous supply of this cofactor $[3,8]$. The $3-\mathrm{HP}$ production pathway from lactic acid is thermodynamically unfavorable [9]. Additionally, this reaction yields a mixture of lactate and 3-HP, a combination that is not suitable for industrial use [9]. Both the malonyl-CoA and the $\beta$-alanine pathways for $3-\mathrm{HP}$ synthesis employ thermodynamically favorable reactions that do not require coenzyme vitamin B12, making these pathways appealing for industrial use [10-13]. Notably, the NADPH-dependent malonyl-CoA reductase (MCR) from Chloroflexus aurantiacus is widely used for 3-HP synthesis. Although MCR catalyzes a two-step reaction of malonyl-CoA to 3-HP via malonate semialdehyde [14], the imbalance of this cascade causes the accumulation of malonate semialdehyde, resulting in a low rate of conversion from malonyl-CoA to 3-HP. Separation of the MCR enzyme into two individual fragments [MCR-C (amino acids 550-1219) and MCR-N (amino acids 1-549)] has been shown to facilitate the rebalancing of the activities of MCR-C and MCR-N, yielding a drastic increase in
3-HP production (3.72 $\mathrm{g} / \mathrm{L}$ in shaking flask cultivation) when expressed in $E$. coli [15].

Using acid-tolerant host cells is one of the most important techniques for making 3-HP production an economical process. A bacterial host such as $E$. coli or $K$. pneumoniae need $\mathrm{pH}$ control during fermentation, and recovery of the desired product subsequently requires acidification of the culture. Acid-tolerant hosts, such as yeasts, have the advantage that the acid form of 3-HP can be produced directly [11]. Notably, budding yeast has been engineered for 3-HP production [16]. However, the growth of $S$. cerevisiae is severely impaired in the presence of $50 \mathrm{~g} / \mathrm{L} 3-\mathrm{HP}$ [16]. Alternatively, Schizosaccharomyces pombe is naturally tolerant to $3-\mathrm{HP}$ and can grow even in the presence of $50 \mathrm{~g} / \mathrm{L} 3-\mathrm{HP}$ [16]. However, there are only a few reports of 3-HP production using $S$. pombe as a host [13].

Improvement of precursor supply is necessary for chemical production. Acetyl-CoA is a precursor of 3-HP and of a wide range of bioproducts, including isoprenoids, fatty acids and lipids, and butanol. Several strategies have been reported for enhancing the level of acetyl-CoA in the yeast cytosol, including introduction of a PDH bypass $[10,18]$, blocking the glyoxylate pathway to decrease acetyl-CoA consumption [19], and enhancing the supply of CoA [20]. In addition, supplying of other key metabolites for bioproduct production can be used to optimize the cultivation process. For instance, the Iriana and Nielsen groups successfully demonstrated 3-HP production using $S$. cerevisiae as a host with defined medium or feed-in-time medium [11]. This feed-in-time medium provides carbon-limited cultivation, possibly because the medium contains high levels of polysaccharides in combination with cellulolytic enzymes, which gradually degrade the polysaccharide and release glucose [11]. The titer of 3-HP on feed-in-time medium was high; however, this $S$. cerevisiae medium has to be specially synthesized and so is not readily available. In contrast, we have developed cell surface-display techniques for this purpose [21-23]. One of our constructs consists of a betaglucosidase (BGL)-displaying $S$. pombe strain; this strain degrades cellobiose and provides glucose as a carbon source, permitting direct growth on cellobiose without the need for enzymatic supplementation [23]. However, there are no reports (to our knowledge) of the production of acetyl-CoA- and malonyl-CoA-derived chemicals using BGL-displaying S. pombe.

The present study aimed to improve the 3 -HP productivity of an engineered $S$. pombe strain via introduction of a malonyl-CoA pathway. To construct such a 3-HPproducing $S$. pombe strain, we used three strategies: (a) balancing the activity between MCR-C and MCR-N, (b) engineering the supply of acetyl-CoA and CoA itself by 
metabolic engineering, and (c) engineering the supply of sugar by cell surface engineering (Fig. 1). Our results demonstrate the potential of $S$. pombe for 3-HP production, showing the potential of cello-oligosaccharides as a raw material for 3-HP production.

\section{Materials and methods}

Strains and media

All strains used in this study are listed in Table 1. S. pombe FY12804 (NBRP) was used as the parental strain. Yeast strains were cultivated in YM medium (BD Diagnostic Systems, Sparks, MD, USA) or EMM medium (ForMedium, Norfolk, United Kingdom) supplemented with glucose or cellobiose as a carbon source.

\section{Plasmid and homologous recombination (HR) donor construction}

Plasmids used in this study are listed in Table 1, and primers are listed in Additional file 1: Table S1. Cas9 and gRNA expression plasmid pMZ374 [24] was purchased from Addgene. A 20-base seed sequence together with the NGG PAM sequence (N20NGG) in the S. pombe genome was selected using CRISPR direct (http://crisp r.dbcls.jp/) [25]. The HR donor sequences used as editing templates were designed to have about 500-bp homology arms to either side (upstream and downstream)

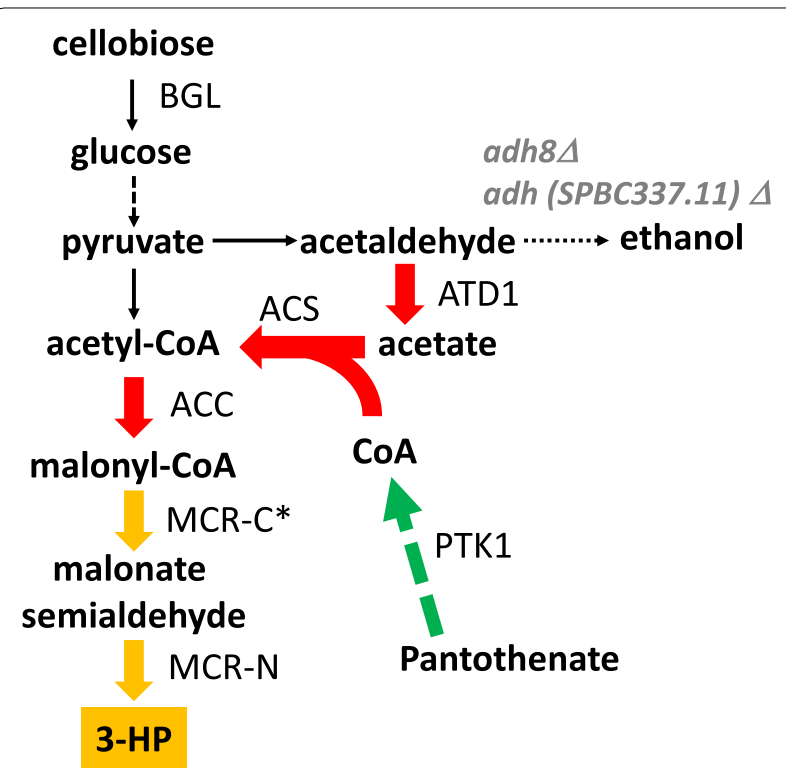

Fig. 1 Engineered metabolic pathway for 3-HP production by S. pombe. Two genes (adh 8 and adh SPBC 337,11 ) were disrupted; the expression of three endogenous genes ( $a t d 1, a c c, p t k 1$ ) was enhanced; and four exogenous genes (acsSE* from Salmonella enterica, separate fragments encoding MCR- $C^{*}$ and MCR-N from Chloroflexus aurantiacus, and BGL from Aspergillus aculeatus) were introduced flanking the Cas9 cutting site and the sites of the intended insertions.

A Cas9 expression plasmid targeting the proteaseencoding aap gene (bp 1-1266) was constructed using the KOD-Plus-mutagenesis Kit (TOYOBO, Co., Ltd., Osaka, Japan) according to the manufacturer's instructions. pMZ374 was used as a template with the primer pair F-aap1-1266+R-aap1-1266. The resultant plasmid was named pMZ374-aap1-1266. Other plasmids for Cas9 targeting were constructed in a similar way and are summarized in Table 1.

To construct the MCR expression cassette, the MCRencoding gene from Chloroflexus aurantiacus was amplified by PCR using codon-optimized synthetic oligonucleotide (Invitrogen) as a template with the primer pair For-MCR + Rev-MCR. The PCR product was cloned into the NheI and SalI sites of pDUAL-FFH1 (RIKEN BRC), and the resultant plasmid was named pDUAL-FFH1MCR. Other plasmids for expression of $m c r-c^{*}$, acc, acs from Salmonella enterica (acsSE*), atd1, and ptk1 were constructed similarly; these plasmids are summarized in Table 1. The PCR templates for fragments encoding $\mathrm{mcr}^{*} \mathrm{c}^{*}$ and acs from S. enterica were obtained as codon-optimized synthetic oligonucleotides (Invitrogen). The PCR products encoding $a c c, a c s S E^{*}$, atd 1 , and $p t k 1$ were cloned (separately) into pDUAL-FFH-31(RIKEN $\mathrm{BRC}$ ); the PCR product encoding $m c r-c^{*}$ was cloned into pDUAL-FFH-61(RIKEN BRC). The PCR product encoding $m c r-n$ was cloned between the NcoI and XhoI sites of pDUAL-hsp-SPBC359.04c_BGL [17]. The resulting plasmid carries $m c r-N$ under the control of the $h s p$ promoter [17].

To construct HR donor DNA for introduction of the acc expression cassette into the chromosomal isp6 region, the acc expression cassette (Pcam-acc-Tadh1) was amplified by PCR with the primer pair For-isp6-1069-3+Revisp6-1069-4. Upstream and downstream regions were amplified with the primer pairs For-isp6-1069-1+Revisp6-1069-2 and For-isp6-1069-5+Rev-isp6-1069-6, respectively. The three amplified fragments were conjugated by overlap extension PCR with the primer pair Forisp6-1069-1+Rev-isp6-1069-6, resulting in HR donor DNA for introduction of the acc expression cassette into the chromosomal isp6 region. Other HR donor DNAs for introduction of fragments encoding $m c r-c^{*}, m c r-n, a c c$, acsSE*, atd1, ptk1, and $b g l$ into the respective genomic regions were constructed similarly; these plasmids are summarized in Table 1.

\section{Genome editing}

All mutagenesis experiments were carried out by cotransformation of $10 \mu \mathrm{L}$ of the pMZ374 vector series with $20 \mu \mathrm{L}$ of the respective PCR product being used as the 
Table 1 Strains and plasmids used in this study

\begin{tabular}{|c|c|c|}
\hline Strain & Genotype & Reference/source \\
\hline 12804 & h90 ura4-D18 ade6-M210 leu1-32 & YGRC/NBRP \\
\hline 12804 ku70 & h90 ura4-D18 ade6-M210 leu1-32 ku704 & Ozaki et al. [17] \\
\hline SPO-01 & 12804 ku $70 \Delta$, isp6 $6 .:$ Pcam 1-acc, integration of $\mathrm{mcr}$ gene at leu & This study \\
\hline SPO-02 & 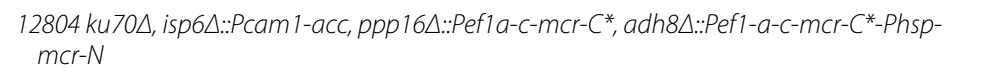 & This study \\
\hline SPO-03 & SPO-02, atg $4 \triangle:: P c a m 1-a c s S E^{*}$, adh $4 \triangle \cdots: P c a m 1$-atd1 & This study \\
\hline SPO-04 & SPO-03, integration of Pcam 1-ptk1 at leu & This study \\
\hline SPO-05 & SPO-04, gut2 $\triangle:: P h s p-S P B C 359.04 c-B G L$ & This study \\
\hline SPO-06 & 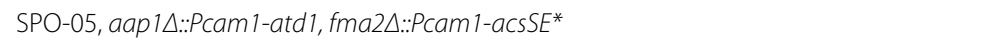 & This study \\
\hline SPO-07 & SPO-06, sxa2A:.Pef1a-c-mcrC* & This study \\
\hline Plasmid & Description & Reference/source \\
\hline pDUAL-FFH31 & Vector under cam 1 promoter control & RIKEN BRC [37] \\
\hline pDUAL-FFH61 & Vector under ef1-a-c promoter control & RIKEN BRC [37] \\
\hline pDUAL-FFH1 & Vector under nmt1 promoter control & RIKEN BRC [37] \\
\hline pDUAL-hsp-SPBC359.04c-BGL & $\begin{array}{l}\text { Vector for cell surface-display expression of protein fusion of BGL from Aspergillus aculea- } \\
\text { tus with SPBC } 359.04 \mathrm{c} \text { anchor protein }\end{array}$ & Ozaki et al. [17] \\
\hline pDUAL-FFH1-MCR & Vector for expression of mcr from Chloroflexus aurantiacus & This study \\
\hline pDUAL-FFH61-MCR-C & Vector for expression of $m c r-C^{*}$ from Chloroflexus aurantiacus & This study \\
\hline pDUAL-hsp-MCR-N & Vector for expression of mcr-N from Chloroflexus aurantiacus & This study \\
\hline PDUAL-FFH31-ACC & Vector for expression of cut6 from Schizosaccharomyces pombe & This study \\
\hline pDUAL-FFH31-ACS & Vector for expression of acsSE* from Salmonella enterica & This study \\
\hline pDUAL-FFH31-ACS1 & Vector for expression of acs 1 from Schizosaccharomyces pombe & This study \\
\hline pDUAL-FFH31-ATD1 & Vector for expression of atd 1 from Schizosaccharomyces pombe & This study \\
\hline pDUAL-FFH31-PTK1 & Vector for expression of ptk1 from Schizosaccharomyces pombe & This study \\
\hline pMZ374 & Vector for expressing adh1:cas9/rrk1:sgRNA in fission yeast: empty sgRNA target & Addgene (\#59896) \\
\hline pMZ374-aap1-1266 & pMZ374 derivative, aap1(1266) sgRNA target & This study \\
\hline pMZ374-atg4-46 & pMZ374 derivative, atg4(46) sgRNA target & This study \\
\hline pMZ374-fma2-950 & pMZ374 derivative, fma2(950) sgRNA target & This study \\
\hline pMZ374-isp6-1069 & pMZ374 derivative, isp6(1069) sgRNA target & This study \\
\hline pMZ374-ppp16-83 & pMZ374 derivative, ppp16(83) sgRNA target & This study \\
\hline pMZ374-sxa2-1436 & pMZ374 derivative, sxa2(1436) sgRNA target & This study \\
\hline pMZ374-adh4-100 & pMZ374 derivative, adh4(100) sgRNA target & This study \\
\hline pMZ374-adh8-434 & pMZ374 derivative, adh8(434) sgRNA target & Ozaki et al. [17] \\
\hline pMZ374-gut2-1749 & pMZ374 derivative, gut2(1749) sgRNA target & Ozaki et al. [17] \\
\hline
\end{tabular}

HR donor DNA for the target locus, as described in a previous report [17]. Briefly, strains were grown in $5 \mathrm{~mL}$ of YM medium at $30{ }^{\circ} \mathrm{C}$ with shaking at $180 \mathrm{rpm}$ until the culture reached an optical density (OD) at $600 \mathrm{~nm}$ of 0.5 ; cells then were co-transformed by electroporation using a Gene Pulser Xcell II (Bio-Rad) and standard methodologies. Transformants were selected using $\mathrm{EMM}+$ Leu plate, then screened by colony PCR and DNA sequencing.

\section{Cultivation and analytical methods}

For 3-HP fermentation from glucose or cellobiose, strains were pre-cultured in $5 \mathrm{~mL}$ YM medium for
3 days at $30{ }^{\circ} \mathrm{C}$ with shaking in a $15-\mathrm{mL}$ test tube, then washed twice with $1 \% \mathrm{NaCl}$ and diluted to an initial OD of 3.0 in $5 \mathrm{~mL}$ EMM medium supplemented with appropriate amino acids and containing glucose $(50 \mathrm{~g} / \mathrm{L})$ or cellobiose $(50 \mathrm{~g} / \mathrm{L}$ or $20 \mathrm{~g} / \mathrm{L})$. The resulting cultures were incubated at $30{ }^{\circ} \mathrm{C}$ with shaking, under aerobic conditions, with sampling at the indicated time points. Cell growth, glucose, and cellobiose levels were analyzed as described previously (Ozaki et al. [17]). 3-HP and acetic acid levels were analyzed using an HPLC equipped with a SCR-102H column $(7 \mu \mathrm{m}$, $8.0 \mathrm{~mm}$ ID $\times 300 \mathrm{~mm}$; Shimadzu) as described previously [17]. 


\section{Fermentations}

SPO-07 was pre-cultured in $5 \mathrm{~mL}$ YM medium for 1 day; the preculture then was inoculated into $100 \mathrm{~mL} \mathrm{YM}$ medium in a 1-L baffled flask and cultured for approximately $24 \mathrm{~h}$. Cells were collected by centrifugation at $900 \mathrm{~g}$ for $10 \mathrm{~min}$ and used to inoculate $400 \mathrm{~mL}$ EMM medium containing cellobiose as a carbon source in a 1-L jar fermenter. Fermentation was performed as follows: agitation rate, $400 \mathrm{rpm}$; temperature, $30{ }^{\circ} \mathrm{C}$; aeration, $1 \mathrm{~L} / \mathrm{min}$ with sterile air; $\mathrm{pH}$ 5.0, maintained by automatic addition of $\mathrm{NH}_{3}(\mathrm{aq})$; dissolved oxygen (DO), 40\%, controlled by agitation. For fed-batch cultivations, the feed was started after $24 \mathrm{~h}$ of cultivation. The feed contained $200 \mathrm{~g} / \mathrm{L}$ cellobiose as a carbon source. The feed rate was set to $5 \mathrm{~mL} / \mathrm{h}$; a total feed volume of $0.2 \mathrm{~L}$ was used per reactor. In the case of glucose was used as a carbon source, initial concentration was $20 \mathrm{~g} / \mathrm{L}$ and the feed contained $200 \mathrm{~g} / \mathrm{L}$ glucose as a carbon source. Other conditions were the same as described above. 3-HP and metabolite analyses were as described above.

\section{Results and discussion}

Reconstruction of a pathway for 3-HP synthesis via malonyl-CoA by coexpression of MCR-C* and MCR-N

To develop a $S$. pombe strain capable of malonyl-CoAmediated production of 3-HP, we introduced two genes: $C$. aurantiacus $m c r$, which encodes malonyl-CoA reductase (MCR); and the endogenous acc (cut6) gene, which encodes acetyl-CoA/biotin carboxylase. MCR converts malonyl-CoA to 3-HP via malonate semialdehyde. ACC has a critical role in both malonyl-CoA supply and fatty acid biosynthesis. Earlier work suggested that strains deficient for intracellular proteases may exhibit improved protein expression [26, 27], a property that might also enhance 3-HP production. In the present work, $m c r$ was introduced by complementation of a Leu- auxotrophy with a leu marker and cut6 (expressed under the control of efla-c constitutive promoter) was introduced by gene replacement at the protease-encoding isp locus. After a 48-h cultivation in EMM medium supplemented with $50 \mathrm{~g} / \mathrm{L}$ glucose, a wild-type $S$. pombe strain did not produce 3-HP to a detectable level, whereas the $m c r$ acc strain (designated SPO-01) generated 3-HP to a titer of $0.016 \mathrm{~g} / \mathrm{L}$ (Fig. 1a).

The $C$. aurantiacus MCR is a bi-functional enzyme that catalyzes a two-step reduction from malonyl-CoA to 3-HP. This enzyme can be separated into two fragments, MCR-C and MCR-N (i.e., the C- and N-terminal subdomains). The MCR-C fragment reduces malonylCoA to malonate semialdehyde, while the MCR-N fragment reduces malonate semialdehyde to 3-HP [15]. The activities of MCR-C and MCR-N are unbalanced, such that malonyl-CoA reduction by MCR-C is the rate-limiting aspect of the two-step reaction [15]. In E. coli, this imbalance was minimized by increasing the expression ratio of MCR-C/MCR-N after saturation mutagenesis of $M C R-C$, yielding a mutant enzyme referred to here as MCR-C*. To recreate the example from $E$. coli, we introduced both fragments [one encoding MCR-N $(m c r N)$ and the second encoding MCR-C* $\left.\left(m c r C^{*}\right)\right]$ into the genome of ACC-expressing $S$. pombe. The resultant strain generated 3-HP to a titer of $0.18 \mathrm{~g} / \mathrm{L}$, which was improved compared to SPO-01 (data not shown). We then introduced an additional copy of $m c r C^{*}$. The resultant strain (SPO02) harbors 2 copies of $m c r C^{*}$ under the control of ef1a$c$ promoter, 1 copy of $m c r N$ under the control of the $h s p$ promoter, and 1 copy of acc under the control of the cam 1 constitutive promoter. SPO-02 generated 3-HP to a titer of $0.53 \mathrm{~g} / \mathrm{L}$ (Fig. 2a), a 30-fold improvement compared to SPO-01. Both strains consumed almost all of the supplied glucose after $24 \mathrm{~h}$, producing $0.5 \mathrm{~g} / \mathrm{L}$ of acetic acid as a byproduct. Introduction of the $m c r N$ and $m c r C *$ fragments did not adversely affect cell growth (Fig. 2d). Introduction of a third copy of $m c r C^{*}$ into SPO-02 did not further enhance 3-HP production (data not shown), suggesting that further improvement of 3-HP titer would require the malonyl-CoA and acetyl-CoA supply.

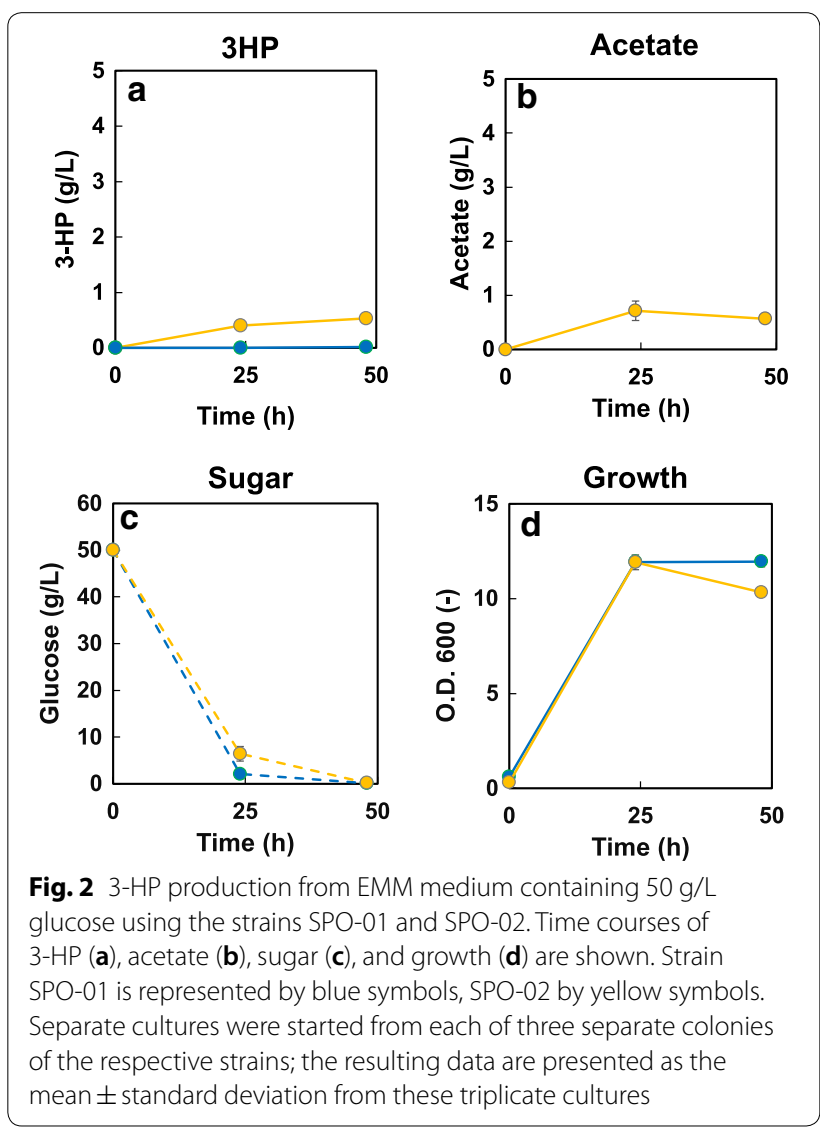




\section{Improvement of 3-HP production by increasing the supply of acetyl-CoA}

To increase the malonyl-CoA supply, we sought to enhance the acetyl-CoA supply. Notably, engineering of the availability of acetyl-CoA has been reported to yield a positive effect on 3-HP production, presumably via the increase in the supply of malonyl-CoA. This strategy has been employed successfully in $S$. cerevisiae to improve the production of 3-HP [11, 28], butanol [29, 30], and isoprenoids [31, 32]. Specifically, these laboratories enhanced the flux towards acetyl-CoA by co-expression of a Salmonella enterica gene encoding acetyl-CoA synthase harboring a point mutation (referred to here as $\operatorname{acs} S E^{*}$ ) and a gene encoding an intact aldehyde dehydrogenase (S. cerevisiae ald6). To apply this approach in $S$. pombe, we introduced acs $S E^{*}$ (under the control of the cam1 promoter) into the protease-encoding atg 4 locus of strain SPO-02. We then introduced a copy of the endogenous atd1 gene (a $S$. pombe homolog of the $S$. cerevisiae ald6 aldehyde dehydrogenase; also under the control of the cam 1 promoter) into the $a d h 4$ locus (which encodes an alcohol dehydrogenase) of the resulting strain. We chose to target an alcohol dehydrogenase-encoding gene based on the observation that the loss of alcohol dehydrogenase activity permits accumulation of acetoaldehyde, a substrate of aldehyde dehydrogenase. However, given a previous report [17] that disruption of the major S. pombe alcohol dehydrogenase locus $a d h 1$ significantly impaired growth, we chose to target the $a d h 4$ locus, which encodes a minor alcohol dehydrogenase.

The resulting SPO-03 strain (which harbors acsSE* and atd1) did not exhibit an improved 3-HP titer (compared to strain SPO-02) (Fig. 3a). However, SPO-03 accumulated acetate to $2.4 \mathrm{~g} / \mathrm{L}$ after $48 \mathrm{~h}$ of cultivation (Fig. 3b). Introduction of additional copies of $\operatorname{acsS} E^{*}$ or of the endogenous acs gene (acs1) could not yield improved 3-HP production (data not shown).

Engineering the CoA supply is an alternative to for improving the acetyl-CoA supply. Pantothenate kinase (PTK) is a key enzyme for improving CoA production [33]. For example, the overexpression of mpanK1b (encoding a mouse PTK homolog) yielded a 13-fold increase of the intracellular CoA concentration in mammalian cells [34]. Similarly, a fivefold increase of acetylCoA levels (with tenfold higher CoA production) was obtained by overexpression of the endogenous PTK in E. coli [35]. In S. cerevisiae, overexpression of the endogenous PTK in combination with the introduction of acs and ald6 was reported to yield $\sim 3.6$-fold increased production of naringenin (a flavonoid) compared to the level obtained upon introduction of acs and ald6 alone [20]. To implement this strategy, we introduced a copy of the endogenous gene encoding pantothenate kinase ( $p t k 1)$
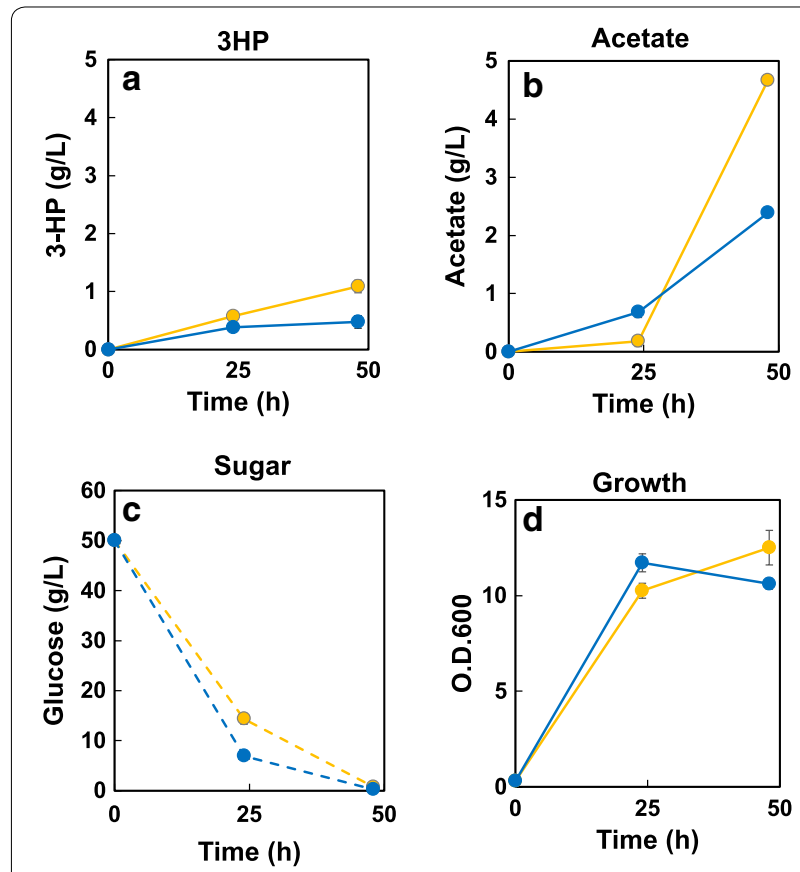

Fig. 3 3-HP production from EMM medium containing $50 \mathrm{~g} / \mathrm{L}$ glucose using the strains SPO-03 and SPO-04. Time courses of 3-HP (a), acetate $(\mathbf{b})$, sugar $(\mathbf{c})$, and growth $(\mathbf{d})$ are shown. Strain SPO-03 is represented by blue symbols, SPO-04 by yellow symbols. Separate cultures were started from each of three separate colonies of the respective strains; the resulting data are presented as the mean \pm standard deviation from these triplicate cultures

into SPO-03 by leu auxotrophic marker. The resultant strain (SPO-04) produced 3-HP of $1.1 \mathrm{~g} / \mathrm{L}$ in after $48 \mathrm{~h}$ cultivation (Fig. 3a), a doubling of titer compared to SPO-03. These results indicated that improving the CoA supply is an effective approach for enhancing the production of malonyl-CoA-derived chemicals. Although intracellular amount of acetyl-CoA could not be detected by acetyl-CoA kit (data not shown), acetate accumulation at $48 \mathrm{~h}$ was increased from $2.4 \mathrm{~g} / \mathrm{L}$ (SPO-03) to $4.7 \mathrm{~g} / \mathrm{L}$ (SPO-04) (Fig. 3b; yellow) in the new strain. The introduction of additional copies of ptk1 did not further improve 3-HP production (data not shown), suggesting that acetyl-CoA is sufficient in strain SPO-04.

\section{Improvement of 3-HP production by controlling the carbon supply}

Avoiding ethanol accumulation is a challenge for 3-HP production in budding yeast. Disruption of the ethanol synthesis pathway is a straightforward approach, but halts growth. Alternatively, 3-HP production under carbon-limited cultivation has been demonstrated in $S$. cerevisiae using feed-in-time medium, which imitates a fed-batch process [12]. This feed-in-time medium 
contains polysaccharides as well as a degrading enzyme, providing a gradual release of glucose. Here, we utilized our previously described cell surface-display technique for producing BGL on the surface of S. pombe [23]. BGL was expressed as a genetic fusion with an anchor protein, SPBC359.04c [23]. The resulting BGL localizes to the cell surface, where the enzyme hydrolyzes cellobiose into glucose. Therefore, we introduced the gene encoding the BGL-anchor fusion protein into SPO-04. The resultant strain (SPO-05) produced 3-HP at $1.0 \mathrm{~g} / \mathrm{L}$ starting from $50 \mathrm{~g} / \mathrm{L}$ of glucose (Fig. $4 \mathrm{a}$ ), a titer similar to that obtained with SPO-04. That is the expression of BGL had no adverse effect on 3-HP production. When $50 \mathrm{~g} / \mathrm{L}$ of cellobiose was used as a carbon source, the BGL-displaying SPO-05 strain produced 3-HP at $1.6 \mathrm{~g} / \mathrm{L}$ after $48 \mathrm{~h}$ of cultivation, a titer was 1.5 -fold that obtained with glucose. These results showed that providing cellobiose (or the cello-oligosaccharide form) as the carbon source permits an on-going release of glucose by gradual degradation via the cell surface-displayed BGL, and cell surface display
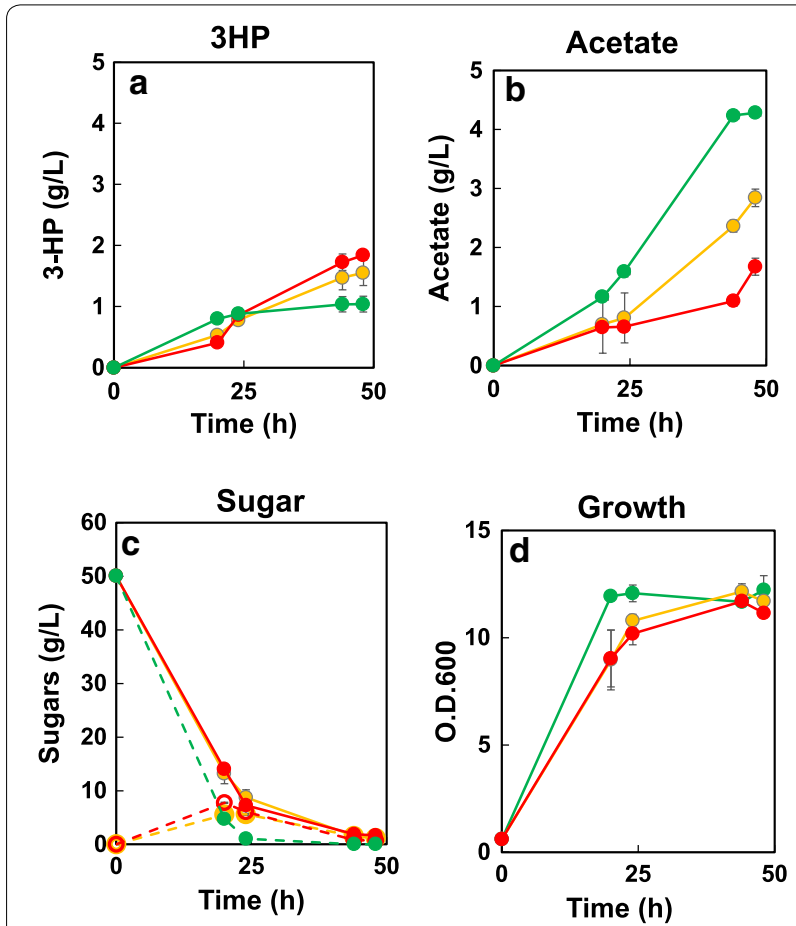

Fig. 4 3-HP production from EMM medium containing $50 \mathrm{~g} / \mathrm{L}$ glucose or cellobiose using the strains SPO-05 and SPO-06. Time courses of 3-HP (a), acetate (b), sugar (c), and growth (d) are shown. In c, dotted lines show glucose and solid lines show cellobiose. Strain SPO-05 grown on glucose is represented by green symbols; SPO-05 grown on cellobiose is represented by yellow symbols; and SPO-06 grown on cellobiose is represented by red symbols. Separate cultures were started from each of three separate colonies of the respective strains; the resulting data are presented as the mean \pm standard deviation from these triplicate cultures was effective for 3-HP production. During growth on glucose, strain SPO-05 consumed $45.3 \mathrm{~g} / \mathrm{L}$ of glucose after $20 \mathrm{~h}$; almost all glucose was consumed by $24 \mathrm{~h}$. After $20 \mathrm{~h}$ of cultivation on cellobiose, cellobiose and glucose were $14 \mathrm{~g} / \mathrm{L}$ and $7.7 \mathrm{~g} / \mathrm{L}$ (respectively) in the medium, corresponding to the consumption of $28.3 \mathrm{~g} / \mathrm{L}$ of cellobiose. The residual amounts of cellobiose and glucose after $24 \mathrm{~h}$ of cultivation were $7.2 \mathrm{~g} / \mathrm{L}$ and $6.0 \mathrm{~g} / \mathrm{L}$, respectively. Thus, SPO-05 cultivated in cellobiose-containing medium appeared to maintain a culture medium glucose concentration of approximately $6-7 \mathrm{~g} / \mathrm{L}$. Cell growth on cellobiose was slightly slower than that on glucose, presumably due to slower glucose supply. Acetate accumulation was notably decreased (from 4.2 to $2.8 \mathrm{~g} / \mathrm{L}$ after $48 \mathrm{~h}$ ) when cellobiose was used as the carbon source. Maintaining a lower glucose concentration in the culture medium by use of BGL display may prevent overflow metabolism, resulting in reducing acetate accumulation [36].

In an attempt to further improve 3-HP production, we introduced additional copies of acc and acsSE* into SPO-05. The resultant strain (SPO-06) produced 3-HP at $1.8 \mathrm{~g} / \mathrm{L}$ of after $48 \mathrm{~h}$ of cultivation, a slight improvement compared to SPO-05. Although SPO-06 exhibited decreased acetate accumulation $(1.6 \mathrm{~g} / \mathrm{L}$ in $48 \mathrm{~h}$ cultivation), 3-HP production was not improved. This observation suggested that an improved malonyl-CoA supply was failed to enhance 3-HP production. Therefore, further improvement of MCR activity is desirable.

\section{Re-tuning of MCR-C expression level}

MCR activity was improved in the SPO-02 strain, and the acetyl-CoA supply then was further strengthened in the SPO-04 strain. By employing cellobiose as a carbon source with BGL display, 3-HP titer was augmented to $1.8 \mathrm{~g} / \mathrm{L}$ in the strain SPO-05. Further enhancement of the flux toward acetyl-CoA decreased acetate accumulation, and the resultant strain SPO-06 exhibited a slightly improved 3-HP titer. Remarkably, SPO-06 exhibited a significantly strengthened malonyl-CoA supply compared to that in SPO-02. Hence, we anticipated that the further augment of MCR activity (to utilize the increased malonyl-CoA supply) might further enhance 3-HP production. Hence, additional copies of the $m c r C^{*}$ gene were inserted into the chromosomal sxa2 locus of SPO-06. It is difficult to evaluate the activities of $\mathrm{mcrC}$ and $\mathrm{mcrN}$ because both enzymes use NADPH as a cofactor. However, enzyme expression was confirmed by SDS-PAGE (Additional file 1: Figure S1A) and qPCR analysis of the resulting strain (SPO-07) revealed that the relative mRNA expression level of $m c r C^{*}$ increased with gene copy number (Additional file 1: Figure S1B). Figure 5 shows that SPO-07, which carries 3 copies of $m c r C^{*}$ in its genome, produced $2.0 \mathrm{~g} / \mathrm{L}$ of $3-\mathrm{HP}$ from $50 \mathrm{~g} / \mathrm{L}$ glucose 

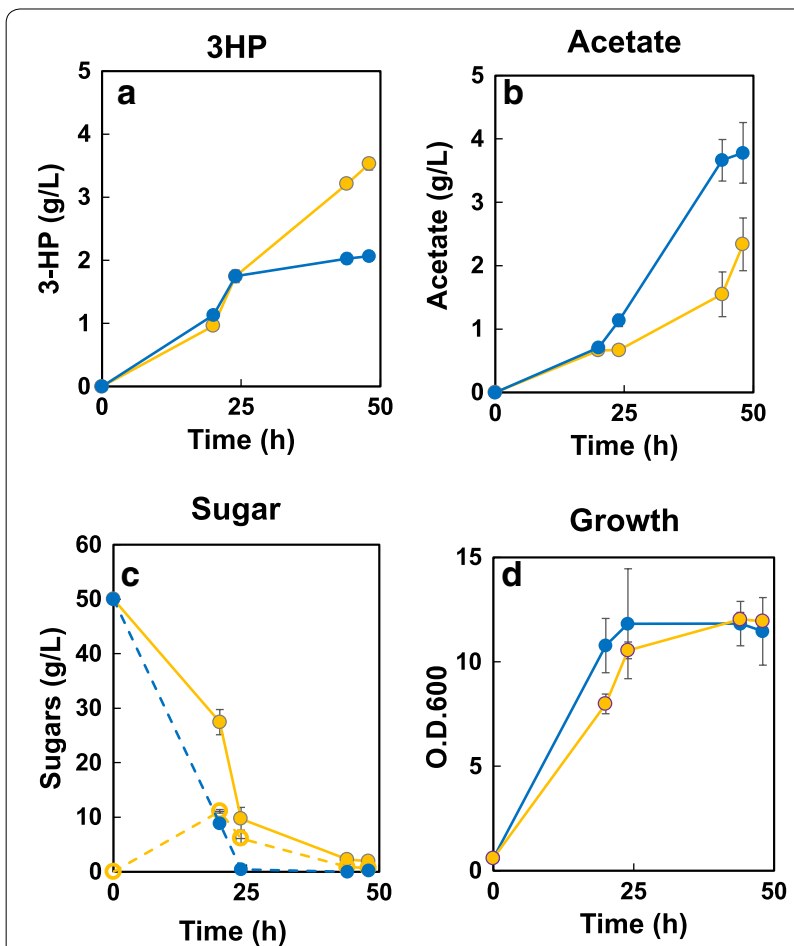

Fig. 5 3-HP production from EMM medium containing $50 \mathrm{~g} / \mathrm{L}$ glucose or cellobiose using strain SPO-07. Time courses of 3-HP (a), acetate (b), sugar (c), and growth $(\mathbf{d})$ are shown. In c, dotted lines show glucose and solid lines show cellobiose. Strain SPO-07 grown on glucose is represented by blue symbols; SPO-07 grown on cellobiose is represented by yellow symbols. Separate cultures were started from each of three separate colonies of the respective strains; the resulting data are presented as the mean \pm standard deviation from these triplicate cultures

after $48 \mathrm{~h}$ of cultivation, a titer twofold higher than that obtained with SPO-05 (1.0 g/L of 3-HP from $50 \mathrm{~g} / \mathrm{L}$ of glucose). When cellobiose $(50 \mathrm{~g} / \mathrm{L})$ was used as the carbon source, SPO-07 produced 3-HP at $3.5 \mathrm{~g} / \mathrm{L}$ after $48 \mathrm{~h}$ of cultivation, a titer twofold higher than that obtained with SPO-06 (as described above). This level corresponded to a yield of $0.063 \mathrm{~g}-3-\mathrm{HP} / \mathrm{g}$-glucose (given that $50 \mathrm{~g}$ cellobiose corresponds to $55.5 \mathrm{~g}$ glucose upon digestion). Introduction of a 4-copies of $m c r-C^{*}$ or 2-copies of $m c r-N$ did not further increase the 3-HP titer (data not shown).

\section{Fed-batch cultivation using cellobiose as a carbon source}

Production of 3-HP under aerobic fed-batch conditions with the limited feeding of cellobiose was evaluated. Specifically, EMM medium supplemented with cellobiose as a carbon source was used. After 24 h of cultivation, when almost all of the original cellobiose was exhausted, further cellobiose feeding was initiated. Under these conditions, strain SPO-07 produced $11.2 \pm 0.6 \mathrm{~g} / \mathrm{L}$ of $3-\mathrm{HP}$ (Fig. 6a) after $96 \mathrm{~h}$ of cultivation, corresponding to a yield of $0.12 \pm 0.007 \mathrm{~g}$ - 3 - $\mathrm{HP} / \mathrm{g}$-glucose. The volumetric production rate in the fed-batch phase was $0.14 \mathrm{~g} / \mathrm{L} / \mathrm{h}$, corresponding to a specific yield of $1.22 \mathrm{~g} / \mathrm{g}$-dry cell weight (DCW). Low levels of acetate and glycerol accumulated under fed-batch culture conditions (Fig. 6b). Glucose concentration increased gradually from the start of feeding, and virtually all of the remaining residual glucose was consumed after feeding was stopped (after $65 \mathrm{~h}$ ). Notably, the results reported here [titer $11.2 \pm 0.6 \mathrm{~g} / \mathrm{L}$, yield $12 \pm 0.7 \%$ (g-3-HP/g-glucose; $1 \mathrm{~g}$ cellobiose is equivalent to $1.1 \mathrm{~g}$ glucose)] are superior to those previously reported for malonyl-CoA-dependent 3-HP production using $S$. cerevisiae. When glucose was used as a carbon source, 3-HP production under fed-batch conditions was also evaluated. After $24 \mathrm{~h}$ cultivation, when all of the original $(20 \mathrm{~g} / \mathrm{L})$ glucose was consumed, further glucose $(200 \mathrm{~g} / \mathrm{L}$ of glucose in feeding medium) feeding was started. Strain SPO-07 also produced $9.2 \pm 0.9 \mathrm{~g} / \mathrm{L}$ of 3-HP (Fig. 6c) after $96 \mathrm{~h}$ of cultivation, corresponding to a yield of $11 \pm 0.11 \mathrm{~g}$-3-HP/g-glucose. Similarly, low levels of acetate and glycerol accumulated under fedbatch culture conditions (Fig. 6d). During feeding phase, ethanol production was kept at low level in spite of glucose accumulation (Fig. 6c, d). Enhancement of glucose uptake may be one of the ways for improving 3-HP titer, as well as shutoff by-products producing pathways. To our knowledge, this work is the first report of chemical production using beta-glucosidase-displaying fission yeast cells under fed-batch condition with cellobiose or glucose feeding.

\section{Conclusions}

In this study, we constructed a series of $S$. pombe strains that produced 3-HP via the malonyl-CoA pathway. 3-HP production was significantly improved through combinatorial strategies including coordination of the expression of MCR-N and MCR-C, overexpression of the acetyl-CoA synthetic pathway, and enhancement of the CoA synthesis pathway. The additional incorporation of BGL display and the use of cellobiose as a carbon source improved 3-HP titer to $11.2 \pm 0.6 \mathrm{~g} / \mathrm{L}$, corresponding to a yield of $12 \pm 0.07 \%$ (g-3-HP/g-glucose; $1 \mathrm{~g}$ cellobiose is equivalent to $1.1 \mathrm{~g}$ glucose) using fed-batch cultivation with cellobiose feeding. These results were superior to the peak 3-HP production previously reported in budding yeast using the malonyl-CoA pathway. Our study 

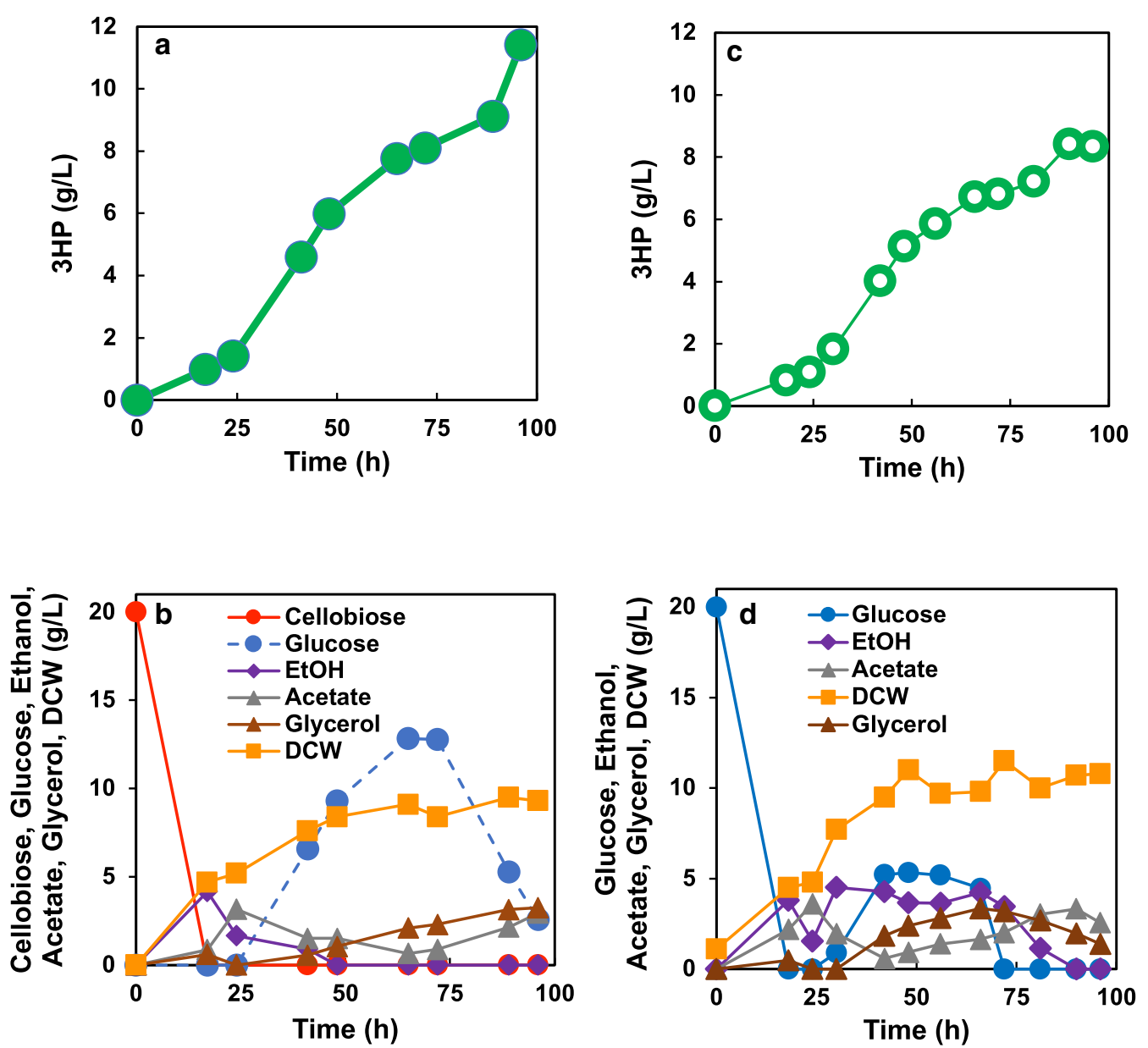

Fig. 6 Fed-batch cultivation of strain SPO-07 using cellobiose $(\mathbf{a}, \mathbf{b})$ or glucose $(\mathbf{c}, \mathbf{d})$ as a carbon source. Time courses of 3-HP $(\mathbf{a}, \mathbf{c})$, other metabolites and dry cell weight (DCW) (b, d) are shown. The concentration of cellobiose (red circles), of glucose generated from cellobiose by cell surface BGL (dotted blue circles), of ethanol (purple triangles), acetate (gray triangles), glycerol (brown triangles), and DCW (orange squares) are shown. In d, glucose concentration is shown solid blue line. The cultivations were performed in triplicate (Additional file 1: Figure S2); representative results are shown here

also demonstrated that BGL display using cellobiose and/or cello-oligosaccharides as a carbon source has the potential to improve the titer and yield of malonyl-CoAand acetyl-CoA-derived compounds.

\section{Additional file}

Additional file 1. Additional figures and tables.

\section{Authors' contributions}

$\mathrm{ST}, \mathrm{AO}$ and TT designed and performed the experiments. RK, CO, MK, and $\mathrm{YH}$. performed the experiments. ST, AO, TM and TT wrote the paper. AK supervised the research. All authors read and approved the final manuscript.

\section{Author details}

${ }^{1}$ Department of Chemical Science and Engineering, Graduate School of Engineering, Kobe University, 1-1 Rokkodaicho, Nada, Kobe 657-8501, Japan.
${ }^{2}$ Graduate School of Science, Technology and Innovation, Kobe University, 1-1 Rokkodaicho, Nada, Kobe 657-8501, Japan.

Competing interests

The authors declare that they have no competing interests.

Availability of data and materials

All data generated or analyzed during this study are included in this published article and its additional information files.

\section{Consent for publication}

Not applicable.

Ethics approval and consent to participate

Not applicable.

\section{Funding}

This work was supported primarily by a Grant-in-Aid for Young Scientists (A) (Grant Number $16 \mathrm{H} 06132$ to TT), and supported in part by Special Coordination Funds for Promoting Science and Technology, Creation of Innovation Centers for Advanced Interdisciplinary Research Areas (Innovative Bioproduction Kobe), MEXT, Japan. 


\section{Publisher's Note}

Springer Nature remains neutral with regard to jurisdictional claims in published maps and institutional affiliations.

Received: 17 April 2018 Accepted: 7 November 2018

Published online: 13 November 2018

\section{References}

1. Karp EM, Eaton TR, i Nogué VS, Vorotnikov V, Biddy MJ, Tan ECD, et al. Renewable acrylonitrile production. Science. 2017;358:1307-10. https:// doi.org/10.1126/science.aan1059

2. Bozell JJ, Petersen GR. Technology development for the production of biobased products from biorefinery carbohydrates-the US Department of Energy's "Top 10" revisited. Green Chem. 2010;12:539-54. https://doi. org/10.1039/B922014C.

3. Kumar V, Ashok S, Park S. Recent advances in biological production of 3-hydroxypropionic acid. Biotechnol Adv. 2013;31:945-61. https://doi. org/10.1016/j.biotechadv.2013.02.008.

4. Dishisha T, Pyo SH, Hatti-Kaul R. Bio-based 3-hydroxypropionic- and acrylic acid production from biodiesel glycerol via integrated microbial and chemical catalysis. Microb Cell Fact. 2015;21(14):200. https://doi. org/10.1186/s12934-015-0388-0.

5. Li Y, Wang X, Ge X, Tian P. High production of 3-hydroxypropionic acid in Klebsiella pneumoniae by systematic optimization of glycerol metabolism. Sci Rep. 2016;27(6):26932. https://doi.org/10.1038/srep26932.

6. Ko Y, Seol E, Sundara Sekar B, Kwon S, Lee J, Park S. Metabolic engineering of Klebsiella pneumoniae J2B for co-production of 3-hydroxypropionic acid and 1,3-propanediol from glycerol: reduction of acetate and other by-products. Bioresour Technol. 2017;244:1096-103. https://doi. org/10.1038/srep26932.

7. Zaushitsyna O, Dishisha T, Hatti-Kaul R, Mattiasson B. Crosslinked, cryostructured Lactobacillus reuteri monoliths for production of 3-hydroxypropionaldehyde, 3-hydroxypropionic acid and 1,3-propanediol from glycerol. J Biotechnol. 2017;10:22-32. https://doi.org/10.1016/j.jbiot ec.2016.11.005.

8. Honjo H, Tsuruno K, Tatsuke T, Sato M, Hanai T. Dual synthetic pathway for 3-hydroxypropionic acid production in engineered Escherichia coli. J Biosci Bioeng. 2015;120:199-204. https://doi.org/10.1016/j.jbios c.2014.12.023.

9. Henry CS, Broadbelt LJ, Hatzimanikatis V. Discovery and analysis of novel metabolic pathways for the biosynthesis of industrial chemicals: 3-hydroxypropanoate. Biotechnol Bioeng. 2010;106:462-73. https://doi. org/10.1002/bit.22673.

10. Chen Y, Daviet L, Schalk M, Siewers V, Nielsen J. Establishing a platform cell factory through engineering of yeast acetyl-CoA metabolism. Metab Eng. 2013;15:48-54. https://doi.org/10.1016/j.ymben.2012.11.002.

11. Borodina I, Kildegaard KR, Jensen NB, Blicher TH, Maury J, Sherstyk S, et al. Establishing a synthetic pathway for high-level production of 3-hydroxypropionic acid in Saccharomyces cerevisiae via $\beta$-alanine. Metab Eng. 2015;27:57-64. https://doi.org/10.1016/j.ymben.2014.10.003.

12. Kildegaard KR, Jensen NB, Schneider K, Czarnotta E, Özdemir E, Klein $\mathrm{T}$, et al. Engineering and systems-level analysis of Saccharomyces cerevisiae for production of 3-hydroxypropionic acid via malonyl-CoA reductase-dependent pathway. Microb Cell Fact. 2016;15:53. https://doi. org/10.1186/s12934-016-0451-5.

13. Suyama A, Higuchi Y, Urushihara M, Maeda Y, Takegawa K. Production of 3-hydroxypropionic acid via the malonyl-CoA pathway using recombinant fission yeast strains. J Biosci Bioeng. 2017;124:392. https://doi. org/10.1016/j.jbiosc.2017.04.015.

14. Liu C, Wang Q, Xian M, Ding Y, Zhao G. Dissection of malonyl-coenzyme A reductase of Chloroflexus aurantiacus results in enzyme activity improvement. PLoS ONE. 2013;8:e75554. https://doi.org/10.1371/journ al.pone.0075554.

15. Liu C, Ding Y, Zhang R, Liu H, Xian M, Zhao G. Functional balance between enzymes in malonyl-CoA pathway for 3-hydroxypropionate biosynthesis. Metab Eng. 2016;34:104-11. https://doi.org/10.1016/j.ymben .2016.01.001.
16. Kildegaard KR, Hallström BM, Blicher TH, Sonnenschein N, Jensen NB, Sherstyk $S$, et al. Evolution reveals a glutathione-dependent mechanism of 3-hydroxypropionic acid tolerance. Metab Eng. 2014;26:57-66. https:// doi.org/10.1016/j.ymben.2016.01.001.

17. Ozaki A, Konishi R, Otomo C, Kishida M, Takayama S, Matsumoto T, et al. Metabolic engineering of Schizosaccharomyces pombe via CRISPR-Cas9 genome editing for lactic acid production from glucose and cellobiose. Metab Eng Commun. 2017;5:60-7. https://doi.org/10.1016/j.meten 0.2017 .08 .002$.

18. Shiba Y, Paradise EM, Kirby J, Ro DK, Keasling JD. Engineering of the pyruvate dehydrogenase bypass in Saccharomyces cerevisiae for highlevel production of isoprenoids. Metab Eng. 2006;9:160-8. https://doi. org/10.1016/j.ymben.2006.10.005.

19. Chen Y, Daviet L, Schalk M, Siewers V, Nielsen J. Establishing a platform cell factory through engineering of yeast acetyl-CoA metabolism. Metab Eng. 2014;15:48-54. https://doi.org/10.1016/j.ymben.2012.11.002

20. Liu W, Zhang B, Jiang R. Improving acetyl-CoA biosynthesis in Saccharomyces cerevisiae via the overexpression of pantothenate kinase and PDH bypass. Biotechnol Biofuels. 2017;10:41. https://doi.org/10.1186/s1306 8-017-0726-z.

21. Tanaka T, Yamada R, Ogino C, Kondo A. Recent developments in yeast cell surface display toward extended applications in biotechnology. Appl Microbiol Biotechnol. 2012;95:577-91. https://doi.org/10.1007/s0025 3-012-4175-0.

22. Tanaka T, Kondo A. Cell surface engineering of industrial microorganisms for biorefining applications. Biotechnol Adv. 2015;2015(33):1403-11. https://doi.org/10.1016/j.biotechadv.2015.06.002.

23. Tanaka T, Matsumoto S, Yamada M, Yamada R, Matsuda F, Kondo A. Display of active beta-glucosidase on the surface of Schizosaccharomyces pombe cells using novel anchor proteins. Appl Microbiol Biotechnol. 2013:97:4343-52. https://doi.org/10.1007/s00253-013-4733-0.

24. Jacobs JZ, Ciccaglione KM, Tournier V, Zaratiegui M. Implementation of the CRISPR-Cas9 system in fission yeast. Nat Commun. 2014;5:5334. https ://doi.org/10.1038/ncomms6344.

25. Naito Y, Hino K, Bono H, Ui-Tei K. CRISPRdirect: software for designing CRISPR/Cas guide RNA with reduced off-target sites. Bioinformatics. 2015;31:1120-3. https://doi.org/10.1093/bioinformatics/btu743.

26. Idiris A, Bi K, Tohda H, Kumagai H, Giga-Hama Y. Construction of a protease-deficient strain set for the fission yeast Schizosaccharomyces pombe, useful for effective production of protease-sensitive heterologous proteins. Yeast. 2006;23:83-99. https://doi.org/10.1002/yea.1342.

27. Idiris A, Tohda H, Sasaki M, Okada K, Kumagai H, Giga-Hama Y, Takegawa K. Enhanced protein secretion from multiprotease-deficient fission yeast by modification of its vacuolar protein sorting pathway. Appl Microbiol Biotechnol. 2010;85:667-77. https://doi.org/10.1007/s00253-009-2151-0.

28. Chen Y, Bao J, Kim IK, Siewers V, Nielsen J. Coupled incremental precursor and co-factor supply improves 3-hydroxypropionic acid production in Saccharomyces cerevisiae. Metab Eng. 2014;22:104-9. https://doi. org/10.1016/j.ymben.2014.01.005.

29. Generoso WC, Schadeweg V, Oreb M, Boles E. Metabolic engineering of Saccharomyces cerevisiae for production of butanol isomers. Curr Opin Biotechnol. 2015;33:1-7. https://doi.org/10.1016/j.copbio.2014.09.004.

30. van Rossum HM, Kozak BU, Pronk JT, van Maris AJA. Engineering cytosolic acetyl-coenzyme A supply in Saccharomyces cerevisiae: pathway stoichiometry, free-energy conservation and redox-cofactor balancing. Metab Eng. 2016;36:99-115. https://doi.org/10.1016/j.ymben.2016.03.006.

31. Yuan J, Ching CB. Mitochondrial acetyl-CoA utilization pathway for terpenoid productions. Metab Eng. 2016;38:303-9. https://doi.org/10.1016/j. ymben.2016.07.008.

32. Tippmann S, Ferreira R, Siewers V, Nielsen J, Chen Y. Effects of acetoacetylCoA synthase expression on production of farnesene in Saccharomyces cerevisiae. J Ind Microbiol Biotechnol. 2017:44:911-22. https://doi. org/10.1007/s10295-017-1911-6.

33. Olzhausen J, Schübbe S, Schüller HJ. Genetic analysis of coenzyme A biosynthesis in the yeast Saccharomyces cerevisiae: identification of a conditional mutation in the pantothenate kinase gene CAB1. Curr Genet. 2009;55:163-73. https://doi.org/10.1007/s10295-017-1911-6.

34. Rock CO, Calder RB, Karim MA, Jackowski S. Pantothenate kinase regulation of the intracellular concentration of coenzyme A. J Biol Chem. 2000;275:1377-83. https://doi.org/10.1074/jbc.275.2.1377. 
35. Vadali RV, Bennett GN, San KY. Cofactor engineering of intracellular CoA/ acetyl-CoA and its effect on metabolic flux redistribution in Escherichia coli. Metab Eng. 2004;6:133-9. https://doi.org/10.1016/j.ymben .2004.02.001.

36. Paczia N, Nilgen A, Lehmann T, Gätgens J, Wiechert W, Noack S. Extensive exometabolome analysis reveals extended overflow metabolism in various microorganisms. Microb Cell Fact. 2012;11:122. https://doi. org/10.1186/1475-2859-11-122.

37. Matsuyama A, Arai R, Yashiroda Y, Shirai A, Kamata A, Sekido S, et al. ORFeome cloning and global analysis of protein localization in the fission yeast Schizosaccharomyces pombe. Nat Biotechnol. 2006;24:841-7. https ://doi.org/10.1038/nbt1222.
Ready to submit your research? Choose BMC and benefit from:

- fast, convenient online submission

- thorough peer review by experienced researchers in your field

- rapid publication on acceptance

- support for research data, including large and complex data types

- gold Open Access which fosters wider collaboration and increased citations

- maximum visibility for your research: over $100 \mathrm{M}$ website views per year

At BMC, research is always in progress.

Learn more biomedcentral.com/submissions 